high professional position. The only difficulty in his case was to secure for him a sufficient rest. It is to be noted that in all these cases the wind is quite inoffensive. Whether it be carbonic acid, common air, hydrogen, or what else I am unable to say, as I have never tested it. By its mode of occurrence, the suddenness and volume of its generation, as well as in its innocence of taste or odour, it is clearly marked off from the foul wind generated by the fermentations of food. It is certainly produced in some way by the agency of the nervous system. Such are the painful symptoms which may be observed and discussed under the head of gastralgia; but a few yet remain.

There is a distension of the body, real or false, which sadly distresses some patients. Women having this distension must, perhaps, go upstairs to remove the corset to obtain ease. The swelling is for the most part real and measurable but I am not prepared to say what is the cause or mode of it. That it is a mere accumulation of wind, I hesitate to suppose. It is often unassociated with any consciousness of the presence of flatulence; and, moreover, it is not confined to the region of the stomach. I am assured by many patients, and their observing friends, that it may extend all over the breast, and even to the neck. It may be vascular, but there is no blush with it.

To the horrible, death-like sinkings which belong to gas tralgia, though not always actually coincident with the pain I have already referred incidentally. No. 80, 1874, a male, aged twenty-eight, after a few months' worry began to haveneuralgic pain in the left arm and up the neck. After this he became subject to nocturnal attacks of "want"-not a craving, or exactly a faintness, but "an awful emptiness, with a dread at the heart." He was substantially quite healthy, and was soon cured on restorative and tonic treatment. I am now seeing a similar case in Leeds, with Mr. Robson, in a neurotic subject. Vertigo is sometimes associated with this symptom. Slighter degrees of this distress often accompany gastralgia or enteralgia, or, with an unaccountable languor, precede them. These subjective sensations are, of course, to be distinguished from the collapse, sometimes alarming in its degree, which is consequent upon the intenser attacks of abdominal neuralgia. The intensity, indeed, of most severe cases of gastro-enteralgia may be seen in the ashen cold face and blue nails. Finally, most gastralgics are subject, not when actually suffering only, but also at all times, to borborygmi. Few people are jgnorant of this phenomenon but it is in neuroties especially that it reaches its most lively and garrulous form. An old and valued domestic, who has recently retired after twenty-five years of household service, and who presented all the phenomena of gastralgia in an active form, was so embarrassed by these audible internal questionings that she almost withdrew herself from waiting at table. In some persons, borborygmi are rhythmical, and coincide with the inspiration. The noise is then rather of a churning character, and, I believe, is made in the stomach only. On examination, it will be found that in these persons the breathing, when full, is wholly abdominal. Even in women the thorax may be motionless. In addition to tonic measures, gentle dumb-bell exercises are most potent to remove this disorder.

And now, gentlemen, have I succeeded in enforcisg that which you already knew-namely, that all the symptoms I have discussed to-day form, not only a consistent, but a very uniform series? Few maladies present a more definite series, or one more capable of rational explanation. Is it not one of the last maladies to be classed with those of irregular, capricious, and fanciful evolution? Shall we then say, as the authors of our text-books do to this moment, that gastralgia is for the most part a malady of hysterical women? If we still say so, then it is indeed time for the lion to paint the man.

WestMinster School For NuRses. - The new building in connexion with this institution was opened on the 19th inst. by the Prince of Wales, who was accompanied by the Princess of Wales. The institution, founded ten years ago, has now been placed upon a permanent footing. The new home will accommodate fifty nurses. The com mittee also hope to establish some district nurses among the poor of Westminster. The cost of the site, including all expenses, is $£ 4939$, which has been paid, but $£ 4000$ is required for the remaining expenses of erection and for furnishing.

\section{ON AN OPERATION FOR THE CORRECTION OF SECONDARY DIVERGENT STRABISMUS.}

BY JOHN TWEEDY, F.R.C.S. ENG,,

PROFESSOR OF OPHTHALMIC MEDICINE AND SURGERY IN UNIVERSITY COLLEGE; SURGEON TO THE ROYAL LONDON OPHTHALMIC IIOSPITAL, MOORFIELDS.

OF the various forms of squint perhaps none is so dis. advantageous to the patient or so troublesome to the surgeon as the divergence that occasionally follows tenotomy of the internal rectus for the correction of convergent strabismus. Not only does the outward position of the cornea give to the countenance a peculiarly sinister expression, but the back. ward displacement of the insertion of the internal rectus muscle renders rotation of the eyeball inwards painful, and perhaps altogether impossible. Binocular vision is no longer attainable, and the sight of the deviating eye steadily declines, till eventually it is practically lost. Hence, the importance of restoring the parallelism of the visual axes as early as possible.

Whenever an eye begins to deviate outwards after tenotomy of the internal rectus, it is certain that there has been what is called "too free division "- not, of course, of the tendon but of the muscular substance, or of the capsule of Tenon Formerly this mishap seems to have occurred more frequently than it does in these days, though I suspect the immunity of later times is due rather to good luck than good manage ment. So long as surgeons aim at correcting convergent strabismus offhand by tenotomy alone, cases of secondary divergence will occur. In operations for squint the rationale of tenotomy seems to be commonly ignored. The modus operandi of tenotomy for remedying other deformities, say club-foot, is not to divide the tendon and then straightway to put the foot in its proper position, but to leave it for awhile in its abnormal situation until reparative material has formed between the ends of the divided tendon. Correc tion is then accomplished by stretching the soft reparative material and thereby elongating the tendon. In operating for squint, on the other hand, both the surgeon and the patient seem bent on immediate rectification of the deformity, and are extremely elated when this is attained. The tendon and adjacent capsule are freely and extensively divided, and then the muscle being liberated from counter-extension con tracts vigorously, so that the severed tendon acquires a new attachment far back and behind the centre of movement 0 the eyeball. Ever afterwards it works at a mechanical dis advantage, and sooner or later exhibits the painful and in effectual efforts of insufficiency. When the eye is "straight" immediately atter tenotomy, it is hailed as "a brilliant success." It is, however, these "brilliant successes" that furnish the largest quota of cases of secondary divergence.

In ordinary non-paralytic squint, when operative measures are called for, it is sufficient to tenotomise the contracted or shortened muscle only-that is, the muscle on that side to which the cornea inclines. In secondary divergence, however, division of the external rectus is not enough. For the reason I have already mentioned, it is necessary, in addition, to bring forward the retracted tendon of the in ternal rectus and attach it to the sclerotic in front of a line passing through the centre of rotation of the eyeball. This proceeding is called Readjustment, and is at once cosmetic and plastic in character.

Many modes of performing readjustment have been sug gested. In this country it is, I believe, usual to employ that which was originally designed by the late Mr. Critchett. It is accomplished in the following way:-

First, the tendon of the external rectus is divided sub. conjunctivally, and then a vertical cut is made with a pair of scissors through the conjunctiva and deep fascia in a line corresponding with the inner margin of the cornea, but at one line and a half to two lines from it. With a few snips the conjunctiva and subjacent fascia are separated from the inner side of the globe as far back as the caruncle, care being taken in doing this to divide the new insertion of the in. ternal rectus so that in drawing the tissues which have been thus detached from the globe the tendon will be raised with them. A vertical slip of the conjunctiva and fascia is then cut away, and the edges of the wound are brought together 
with sutures. Three threads are required, the centre one being inserted first ; it is passed through the slip of conjunctiva which was left at the edge of the cornea, and then through the deep fascia and conjunctiva close to the caruncle. The upper and lower stitehes are then put in and tied. ${ }^{1}$

I have never attempted readjustment by the Critchett method, because, so far as I have had opportunity of judging it as performed by others, it seems somewhat haphazard in its processes, and not very satisfactory in its results. doubt not that in many instances it has been perfectly successful, I speak only of the cases I have myself witnessed; of four such only one could be said to be perfect; of the remaining three one had a prominent hard cicatricial band in the conjunctiva, another had permanent displacement upwards and outwards of the cornea from rotation, and in the third there was absolute immobility of the globe from cellulitis of the orbit following the operation. The faults of the method are threefold : first, the primary division of the external rectus favours rotation and other disadvantageous displacements of the globe, which are further increased when the internal rectus is cut through; second, the internal rectus is divided without any precaution being taken to establish its identity, so that when in the later stages of the operation it is sought to affix the sutures there is no certainty that what is secured is solely or wholly muscle, or even muscle at all ; and third, there is an unnecessary amount of exposure of the internal rectus muscle and orbital cellular tissue.

Impressed with the many defects of this operation, I began to think of how they might be avoided. At length the plan described below suggested itself. Five years and a half ago I put it in practice for the first time, and since then I have repeated it about fifteen times. Speaking generally, the processes of my method are exactly the reverse of those of the late Mr. Critchett's. What he did first I do last, and what he did last I do first. There are, however, other differences, as will be observed in the following description, whereby greater certainty and security are obtained with less risk

The operation, then, consists of ten stages, as follows:-

1. The lids being kept apart with a speculum, a fine silk thread is inserted into the conjunctiva and episcleral tissue, in a line with the horizontal diameter of the cornea, and about one-sixteenth of an inch from the inner margin of the cornea. The ends of the thread are left long with the needle attached (see Fig. 1). (The preliminary insertion of

FIG.

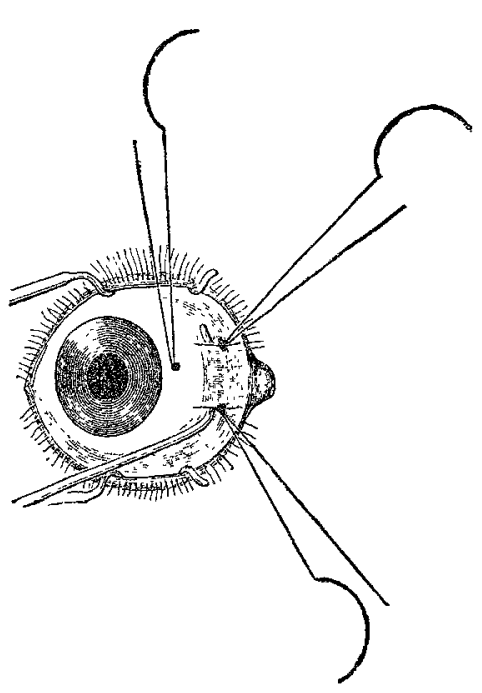

this thread marks, and afterwards indicates, the horizontal meridian of the eyeball, and still later serves as a suture for the middle of the rectus muscle.)

2. Next, a crescentic incision is made immediately to the nasal side of the thread and through the conjunctiva only.

3. The conjunctiva is then gently detached from the underlying capsule towards the caruncle, but only far enough to expose the insertion of the rectus muscle; not to strip the muscle.

4. A strabismus hook is then passed under the muscle. 5. While the hook is in position fine silk threads are attached to the upper and lower borders of the muscle and left long (see Fig. 1).

1 Lawson: Diseases and Injuries of the Eje, fourth edition, p. 262.
6. The hold of the threads having been tested by gentle traction, the muscle is divided on the hook. (The operator is now sure that he has secured the tendon.)

7. The muscle is then carefully raised by means of the threads, and any remaining attachments of the muscle to the globe completely divided.

8. The needle attached to the thread at the inner border of the cornea is passed through the middle of the divided muscle from within outwards, and in such a way as to penetrate the muscle and its sheath and overlying conjunctiva. (By including the sheath and conjunctiva, not only is a firmer attachment obtained, but the relations of the muscle and its sheath are as far as possible preserved, at the same time that the sunken caruncle is raised by bringing the conjunctiva forwards.)

9. The needles attached to the threads at the upper and lower borders of the muscle are now insinuated into the episcleral tissue, and made to emerge on the surface of the con junctiva at about one-eighth of an inch from the upper and lower margins of the cornea respectively (see Fig. 2). This gives a broad and fan-like attachment to the muscle.

FIG. 2.

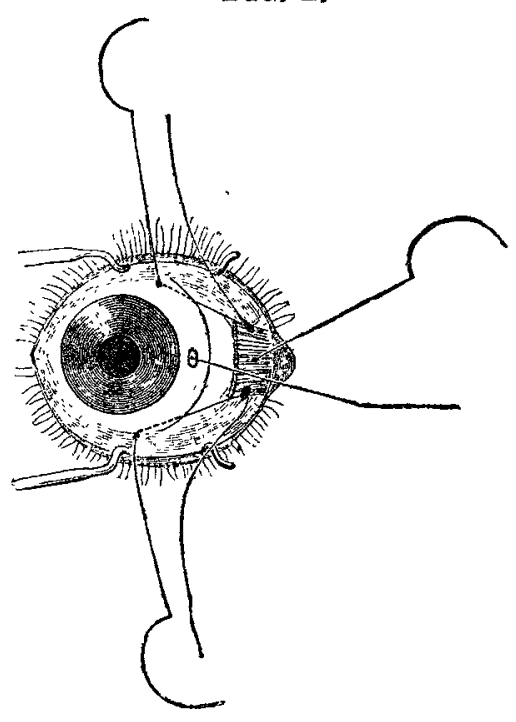

10. The external rectus is now freely divided subconjunctivally, and then, while an assistant rotates the eyeball inwards, the corresponding ends of the three sutures are closely and firmly tied and cut short.

Should shortening of the internal rectus muscle or removal of redundant conjunctiva be deemed necessary, it may be done just before the ninth stage. I have, however, only once found it necessary to abscise any muscular substance. As a rule the muscle is already short enough; it only needs its attachments to be brought forward. To ensure a complete and permanent result, there should be some convergence of the eye immediately after the operation, and no attempt should be made to exercise the muscle for a week afterwards. The dressing and after-treatment are simple. After washing the lids with a weak solution of boracic acid or of chloride of sodium (about 1 per cent.), I apply to the closed lids a piece of lint on which a thin layer of boracic acid ointment (gr. 20 ad 5 1) has been spread, and then tie up the eye with a light compress of cotton-wool. The bathing and the compresses are renewed once or twice a day for five or six days. The stitches are removed on the fourth or fifth day if they do not fall of themselves.

Harley-street, $\mathbf{w}$.

Fatality at Leicester County Lunatic Asylum. At the post-mortem examination of the body of a lunatic who recently died at this institution, it was discovered that four of the right ribs and one of the left were broken, the latter having an abscess behind it. No information could be obtained as to how the injuries were inflicted. Further inquiries being instituted, an attendant recollected that $a$ scuffle had taken place between the deceased and another patient, named Burrows, about three weeks previously. During the struggle Burrows and a heavy fire-guard fell on the deceased, who fainted and lay on the floor some time. The attendant did not report the circumstance, and forgot all about it. The jury returned a verdict that deceased died from injuries accidentally caused by Burrows and the fireguard falling upon him 